\title{
Evaluation of autologous skeletal muscle-derived factors for regenerative medicine applications
}

\section{Yoshikawa, \\ T. Nakasa, \\ M. Ishikawa, \\ N. Adachi, \\ M. Ochi}

Institute of Biomedical \& Health Sciences, Hiroshima University, Japan
- M. Yoshikawa, MD, Clinical Staff,

- T. Nakasa, MD, PhD, Assistant Professor,

M. Ishikawa, MD, PhD,

Assistant Professor,

N. Adachi, MD, PhD, Professor

n. Mchi, MD, PhD, Emeritus

President, Department of

Orthopaedic Surgery, Institute

of Biomedical \& Health Sciences,

Hiroshima University, Japan.

Correspondence should be sent to T. Nakasa;

email: tnakasa0@gmail.com

doi: $10.1302 / 2046-3758.65 . B J R-$ 2016-0187.R1

Bone Joint Res 2017;6:277-283.

Received: 01 August 2016;

Accepted: 18 November 2016

\section{Objectives}

Regenerative medicine is an emerging field aimed at the repair and regeneration of various tissues. To this end, cytokines (CKs), growth factors (GFs), and stem/progenitor cells have been applied in this field. However, obtaining and preparing these candidates requires invasive, costly, and time-consuming procedures. We hypothesised that skeletal muscle could be a favorable candidate tissue for the concept of a point-of-care approach. The purpose of this study was to characterize and confirm the biological potential of skeletal muscle supernatant for use in regenerative medicine.

\section{Methods}

Semitendinosus muscle was used after harvesting tendon from patients who underwent anterior cruciate ligament reconstructions. A total of 500 milligrams of stripped muscle was minced and mixed with $1 \mathrm{~mL}$ of saline. The collected supernatant was analysed by enzymelinked immunosorbent assay (ELISA) and flow cytometry. The biological effects of the supernatant on cell proliferation, osteogenesis, and angiogenesis in vitro were evaluated using human mesenchymal stem cells (hMSCs) and human umbilical cord vein endothelial cells (HUVECs).

\section{Results}

The supernatant contained several GFs/CKs, with especially high levels of basic fibroblast growth factor, and CD34+ cells as the stem/progenitor cell fraction. With regard to biological potential, we confirmed that cell proliferation, osteoinduction, and angiogenesis in hMSCs and HUVECs were enhanced by the supernatant.

\section{Conclusions}

The current study demonstrates the potential of a new point-of-care strategy for regenerative medicine using skeletal muscle supernatant. This attractive approach and readily-available material could be a promising option for tissue repair/regeneration in the clinical setting.

Cite this article: Bone Joint Res 2017;6:277-283.

Keywords: Muscle, Cytokine, Growth factor

\section{Article focus}

- The potential of muscle as a new approach of the regenerative medicine was evaluated.

- Supernatant from minced muscle in saline was analysed as the candidate for tissue repair / regeneration in point-ofcare approach.

- Cell characterisation, the ability of osteogenesis and angiogenesis in supernatant of muscle were analysed.

\section{Key messages}

- Supernatant of muscle included CD34+ and CD44+ cell fractions and several growth factors, especially bFGF.
Supernatant of muscle accelerates cell proliferation, osteogenic differentiation and angiogenesis.

- These results suggest that supernatant of muscle can be applied regenerative medicine in easy way.

\section{Strengths and limitations}

- Strength: This study shows the effect of supernatant muscle obtained in easy way on tissue repair / regeneration.

- Limitations: The therapeutic trials in vivo animal studies were not performed. 


\section{Introduction}

Regenerative medicine is an emerging field aimed at the repair and regeneration of various tissues. A range of materials, such as stem/progenitor cells, cytokines (CKs) and growth factors (GFs), have been utilised as feasible and accessible candidates for tissue repair and regeneration, and their efficacy has been confirmed ${ }^{1}$. However, the procurement and preparation of these factors involves invasive, costly, and time-consuming procedures ${ }^{2}$. Therefore, we adopted the concept of a point-of-care approach as a simpler and more favourable option for use in the daily clinical setting.

Previous studies have demonstrated that skeletal muscle contains various factors such as stem/progenitor cells and CKs/GFs. ${ }^{3-5}$ Moreover, several reports have shown comparable healing of cartilage and bone treated with muscle-derived cells (MDCs). These facts suggest that skeletal muscle contains cells that are favourable for musculoskeletal tissue repair. ${ }^{6-8}$ It has been reported that skeletal muscle mass makes up approximately $35 \%$ of body weight, therefore we hypothesised that skeletal muscle could be a feasible target to obtain a patient's autologous tissue as a biological source for tissue repair with a pointof-care approach. ${ }^{9}$ In this study, we tested the hypothesis that various factors, including stem/progenitor cells and CKs/GFs, would be easily obtained from skeletal muscle supernatant and that this supernatant would have therapeutic potential.

The purpose of this study was to characterise the cell population and the amounts of CKs and GFs in musclederived supernatant obtained via a point-of-care approach and to investigate its angiogenic and osteogenic potentials for future clinical applications in the field of orthopaedic surgery.

\section{Materials and Methods}

This study was approved by the Ethics Committee of the Graduate School of Biomedical Sciences, Hiroshima University.

Human skeletal muscle and preparation of supernatant from muscle tissue. Skeletal muscle from the semitendinosus tendon was harvested from a total of ten patients who underwent anterior cruciate ligament (ACL) reconstruction. Stripped muscle was minced in the operating room, and then transferred to the bench for further processing. Minced tissue, $500 \mathrm{mg}$ of each sample, was mixed well with $1 \mathrm{~mL}$ of saline and centrifuged at $900 \mathrm{rpm}$ for two minutes. The supernatant was collected, transferred to a new tube and analysed as described below. The number of samples available for each experiment varied on the size of the available muscle and therefore the amount of supernatant available from the individual specimen processing.

Experimental design. Two experiments were performed in this study. First, to characterise the supernatant from skeletal muscle that presumably contains growth factors, cytokines and cells, enzyme-linked immunosorbent assay (ELISA) and flow cytometric analyses were used. For the ELISA, vascular endothelial growth factor (VEGF), insulin-like growth factor-1 (IGF-1), basic fibroblast growth factor (bFGF), and stromal cell-derived factor-1 (SDF-1) were evaluated. For detection and characterisation of the cell fraction in skeletal muscle supernatant, the sample was stained with the stem/progenitor cell markers CD34, CD44, CD45 and CD105, and analysed by flow cytometry.

Second, the biological potential of muscle supernatant was evaluated in vitro. To confirm the effect on cell proliferation, we added supernatant from muscle to human mesenchymal stem cells (hMSCs) and evaluated proliferation using a cell-growth assay at days one and three. Osteoinductive and angiogenic potentials of muscle supernatant were investigated using hMSCs and the tube formation assay with human umbilical vein endothelial cells (HUVECs).

ELISA analysis. VEGF, IGF-1, bFGF and SDF-1 were quantitatively evaluated by ELISA using the Quantikine system (R\&D Systems Inc., Minneapolis, Minnesota). According to the manufacturer's protocol, standards and pretreated samples were pipetted into the 96 wells. After washing away any unbound substances, an enzyme-linked polyclonal antibody specific for each growth factor was added to the wells. Following a wash to remove any unbound antibody-enzyme reagent, a substrate solution was added to the wells and colour developed in proportion to the amount of growth factor bound in the initial step. The colour development was stopped and the intensity of the colour was measured using a microplate reader set to $450 \mathrm{~nm}$. The obtained values were used to calculate the target protein concentration from a standard curve.

Flow cytometric analysis. Flow cytometry was conducted using the skeletal muscle supernatant. Specific antibodies were used for cell labelling: CD34 antibody for stem/ progenitor cells, CD44 and CD105 antibodies for MSCs, and CD45 antibody for haematopoietic lineage cells (all from BD Biosciences, Franklin Lakes, New Jersey). Samples underwent regular flow cytometric profiling with a FACSCalibur Analyser and CellQuest Pro Software (BD Biosciences Immunocytometry Systems, San Jose, California). Dead cells were excluded from the plots on the basis of propidium iodide (PI) staining (Sigma-Aldrich Corp., St Louis, Missouri). Cells were washed twice with Hanks' balanced salt solution (HBSS) containing 3.0\% heat-inactivated foetal bovine serum (FBS) and incubated with monoclonal antibodies for 30 minutes at $4{ }^{\circ} \mathrm{C}$ after Fc recepter blocking reagent (Miltenyi Biotec $\mathrm{GmbH}$, Bergisch Gladbach, Germany). The stained cells were washed three times with $\mathrm{HBSS} / 3.0 \% \mathrm{FBS}$, resuspended in $0.5 \mathrm{~mL}$ of $\mathrm{HBSS} / 3.0 \% \mathrm{FBS} / \mathrm{PI}$, and analysed. The following monoclonal antibodies were used to identify the 
CD34+/CD45dim subpopulation (the stem/progenitor cell fraction), and the CD44+/CD105+ subpopulation (the MSC fraction): CD34-FITC (clone RAM34), CD44APC (clone G44-26), CD105-PE (clone 266), CD45-FITC (clone HI30), IgG1-PE isotype control, and IgG1-FITC (all from BD Biosciences Pharmingen San Diego, California).

Cell culture. Primary human bone marrow mesenchymal stem cells (hBMMSCs; Life Technologies Corp., Carlsbad, California) were cultured in StemPro MSC SFM (Life Technologies) with antibiotics (at a final concentration of $100 \mathrm{units} / \mathrm{mL}$ penicillin, $100 \mu \mathrm{g} / \mathrm{mL}$ streptomycin and $0.25 \mu \mathrm{g} / \mathrm{mL}$ amphotericin B; Nacalai Tesque, Kyoto, Japan) in humidified air containing $5 \% \mathrm{CO}_{2}$ at $37^{\circ} \mathrm{C}$. Cultured and passaged MSCs were utilised for experiments as described below.

Cell-growth assay. Cell-growth assay was performed using Cell Counting Kit-8 (CCK-8; Dojindo Laboratories, Kumamoto, Japan). Cultured MSCs, passage 3, were inoculated into a 96 -well plate (2000 cells/well). We then added $5 \mu \mathrm{L}$ phosphate buffered saline (PBS) to wells of the control group $(n=10)$ and $5 \mu \mathrm{L}$ muscle supernatant to the muscle supernatant group $(n=10)$ on day 0 with pre-incubation performed for 24 or 72 hours in a humidified incubator. Substrate (highly water-soluble tetrazolium salt, WST-8) was then delivered into each well, and after two hours of incubation the absorbance was measured at $450 \mathrm{~nm}$ using a microplate reader.

Osteoinductive culture of MSCs and evaluation. For osteogenic induction, cells were cultured to $80 \%$ confluence in a 24-well plate, and the medium was replaced with osteogenic induction medium using the StemPro osteogenesis differentiation kit (Life Technologies, Grand Island, New York). At three, six, nine, and 12 days after induction of osteogenesis, the medium was changed and $10 \mu \mathrm{L}$ of PBS or $10 \mu \mathrm{L}$ muscle supernatant was added to the cultured dishes in each group. All experiments were carried out in triplicate. After 14 days of osteoinduction, gene expression was analysed by real-time polymerase chain reaction (RT-PCR) and cultures were stained with alizarin red. Runt-related transcription factor 2 (RUNX2), collagen type I alpha 1 (COL1A1) and osteocalcin were used as bone-related genes. Total RNA was isolated using TRIzol reagent (Invitrogen Corp., Carlsbad, California) and total RNA yields were calculated and quality was determined using absorption spectrochemical analysis. CDNA was synthesised using $2 \mu \mathrm{g}$ of total RNA with a SuperScript VILO kit (Invitrogen) according to the manufacturer's protocol. RT-PCR was performed using the following TaqMan gene expression assay probes (Life Technologies): RUNX2 (Hs00231692_m1), COL1A1 (Hs01076777_m1), osteocalcin (Hs01587814_g1), and glyceraldehyde 3-phosphate dehydrogenase (GAPDH) (Hs02758991_m1). The GAPDH gene was used as a control to normalise differences in total RNA levels in each sample. A threshold cycle (CT) was observed in the exponential phase of amplification, and quantification of relative expression levels was performed using standard curves for target genes and the endogenous control. Geometric means were used to calculate the delta-delta $C T(\Delta \Delta C T)$ values and results are expressed as $2-\Delta \Delta C T$. The value of each control sample was set at 1 and the mean biological duplicate of each sample was used to calculate the fold-change of target genes.

For qualitative analysis of bone formation, alizarin red staining was performed. ${ }^{10}$ Aliquots of $1.0 \mathrm{~mL}$ of $10 \%$ formalin were added to each well and cells were fixed for 15 minutes at room temperature. The formalin solution was then removed, and $500 \mu \mathrm{L}$ of $40 \mathrm{mM}$ alizarin red stain ( $\mathrm{pH} \mathrm{4.1)} \mathrm{was} \mathrm{added} \mathrm{to} \mathrm{each} \mathrm{well.} \mathrm{The} \mathrm{plates} \mathrm{were}$ incubated at room temperature for 20 minutes with gentle shaking, after which the dye solution was pipetted out and the stained monolayers were visualised by microscopy.

Tube formation assay. Angiogenesis is the process of generating new capillary blood vessels. We examined the effects of muscle supernatant on angiogenic activity and tube formation of human umbilical vein endothelial cells (HUVECs). HUVECs were purchased from Lonza Group AG (Basel, Switzerland) and pre-cultured with endothelial basal medium-2 (EBM-2) (Lonza, Basel, Switzerland) and then reseeded at a density of $1.5 \times 10^{4}$ cells/well in 96-well plates pre-coated with Matrigel Matrix (Merck Millipore, Billerica, Massachusetts). We then added 5 $\mu \mathrm{L}$ of muscle supernatant to the supernatant group and incubated at $37^{\circ} \mathrm{C}$ overnight in a tissue-culture incubator. All experiments were carried out in triplicate. We investigated total tube length at 12 and 24 hours by means of Image J analysis of digital images according to the method described in previous reports. ${ }^{11}$

Statistical analysis. All data are expressed as mean and standard deviation (SD). The Mann-Whitney $U$ test was used to detect differences between the two groups. A p-value less than 0.05 was considered statistically significant.

\section{Results}

ELISA analysis revealed that skeletal muscle supernatant contained detectable amounts of CKs and GFs (VEGF $3.80 \mathrm{pg} / \mathrm{mL}$, SD 8.16 , SDF-1: $3.58 \mathrm{pg} / \mathrm{mL}$, SD 8.50 , bFGF: $325.6 \mathrm{pg} / \mathrm{mL}$, SD 210.2 , IGF-1: $82.0 \mathrm{pg} / \mathrm{mL}$, SD 58.2 ). Flow cytometric analysis demonstrated the presence of CD34+ and CD44+/CD105+ cells as the stem/progenitor cell fraction, although the actual number of cells in the muscle supernatant was very small (Fig. 1).

Cell-growth assay at 24 and 72 hours after addition of muscle supernatant revealed that cell growth was significantly increased compared with the PBS group at both time points (Fig. 2).

In the muscle supernatant group, alizarin red staining at 14 days showed larger stained areas compared with 


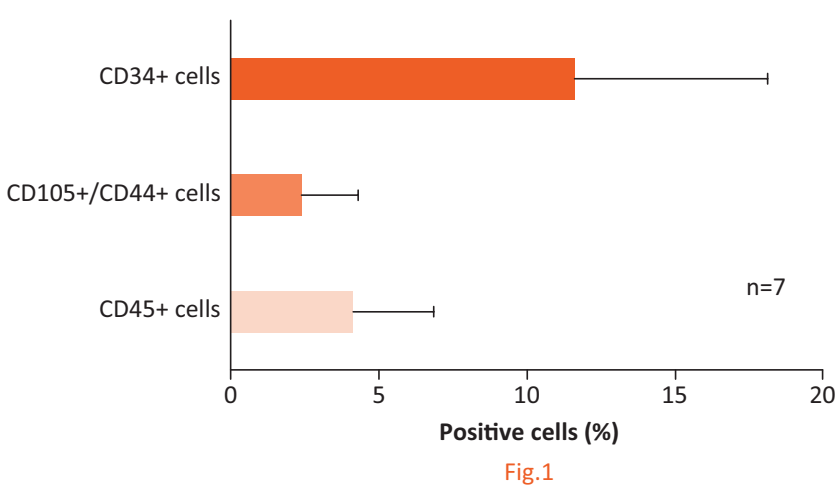

Flow cytometric analysis of supernatant from skeletal muscle $(n=7)$. The stem/progenitor cell fraction, CD34+ and CD105+/CD44+ cells, was confirmed. The presence of cells of the haematopoietic lineage, CD45+ cells, was also demonstrated.

the PBS group (Fig. 3a). Moreover, the expression levels of RUNX2, COL1a1, and osteocalcin were significantly upregulated in the muscle supernatant group compared with the PBS group (all $p<0.05$, Fig. 3b).

The tube formation assay showed that the total tube length was significantly greater in the muscle supernatant group compared with the PBS group at both the 12 and 24 hour time-points ( $p<0.05$ for both) (Fig. 4).

\section{Discussion}

In this study, we showed that the supernatant from skeletal muscle contains various proteins and cell types and has favourable biological effects on cell proliferation, osteoinduction and angiogenesis. To our knowledge, this is the first report to demonstrate the therapeutic potential of skeletal muscle supernatant for tissue repair, aiming for future clinical application following the point-of-care concept.

Many factors such as surrounding tissues, blood supply, nutrient delivery, biomechanical forces, and the supply of several growth factors can affect tissue healing. In an attempt to provide these conditions, many approaches have been applied, ${ }^{12-14}$ however, a time-saving, low-cost and less invasive approach is desirable in practical clinical settings. Hence, the concept of point-of-care has been widely accepted and applied in the field of orthopaedic surgery such as platelet-rich plasma (PRP) administration and fibrin clot delivery for tissue repair and regeneration. ${ }^{15,16}$

It is reported that skeletal muscle releases abundant CKs and GFs following traumatic injury ${ }^{11}$ and also that muscle-derived stem cells have great potential to maintain and contribute to the repair and regeneration of muscle tissue. In addition to this biological potential, the anatomical distribution of skeletal muscle in the human body makes it easy to obtain skeletal muscle tissue from a
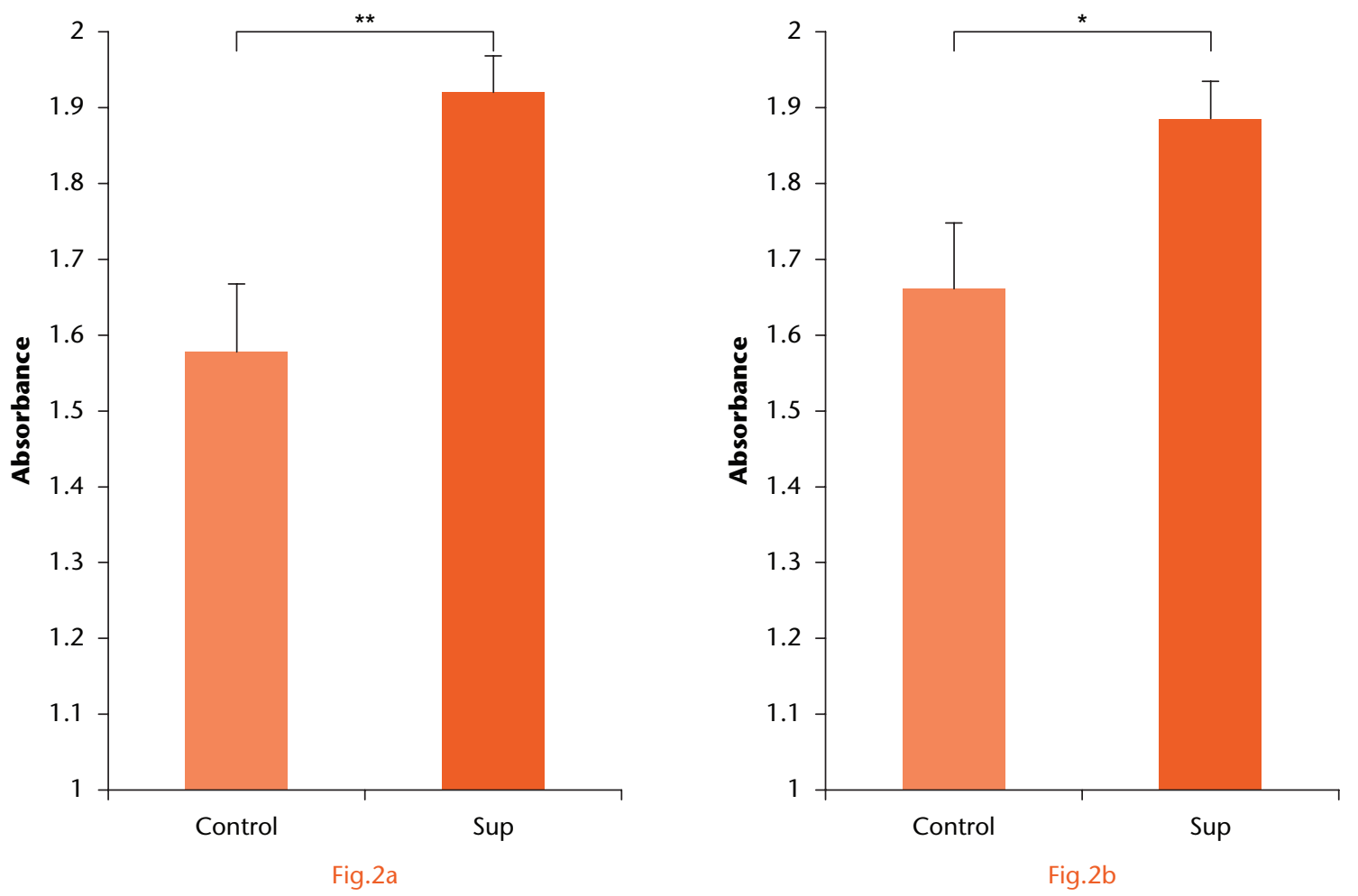

Cell-growth assay (WST-8) at 12 hours (a) and at 24 hours (b) after the addition of skeletal muscle supernatant ( $n=10)\left(* ; p<0.01,{ }^{* *} ; \mathrm{p}<0.05\right)$. The PBS group was treated with $5 \mu \mathrm{L}$ PBS and the Supernatant group was treated with $5 \mu \mathrm{L}$ of muscle supernatant $(\mathrm{n}=10)$. Data are shown as means and standard deviation (*; $\left.p<0.01,{ }^{* *} ; p<0.05\right)$. The asterisks indicate that cell growth in the group treated with skeletal muscle supernatant was significantly higher than that of the PBS-treated group both at 24 and 72 hours. 

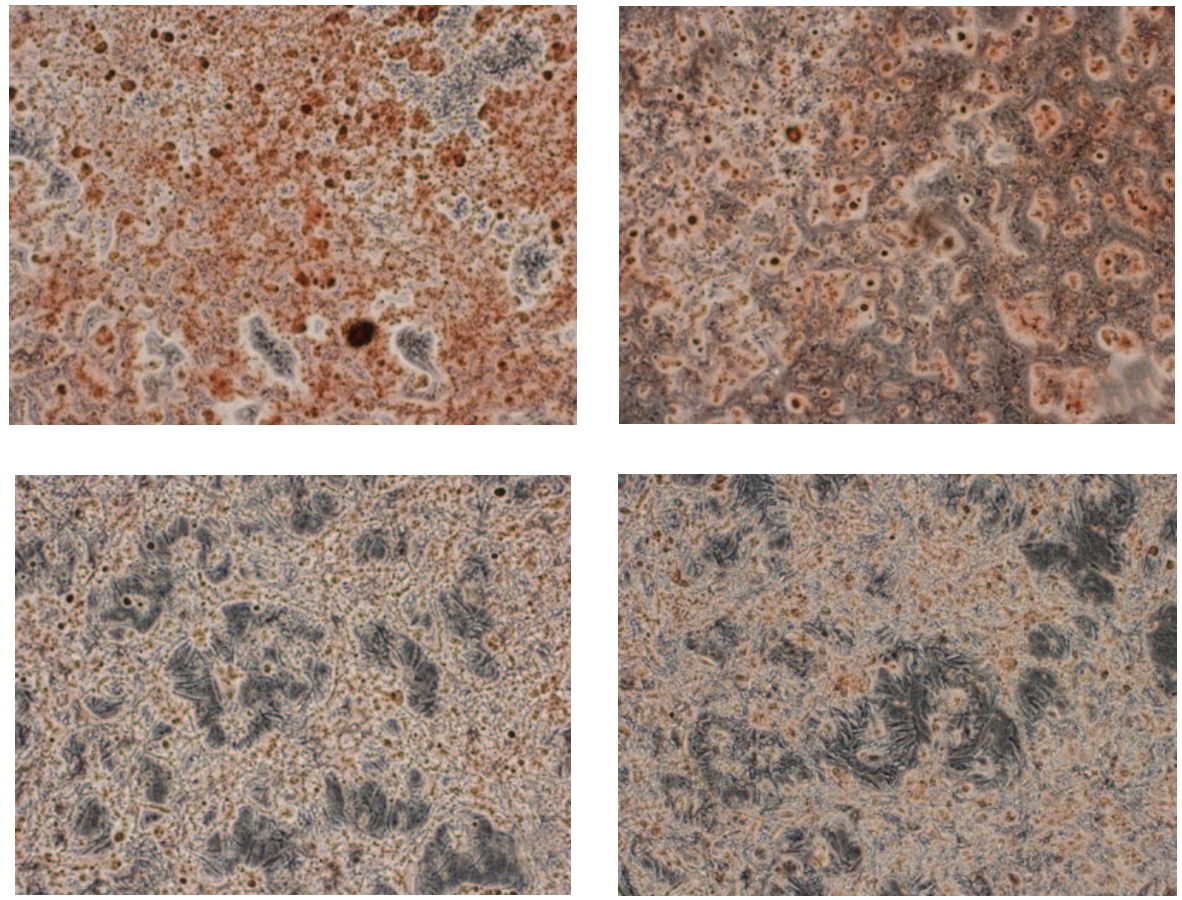

Fig. $3 a$
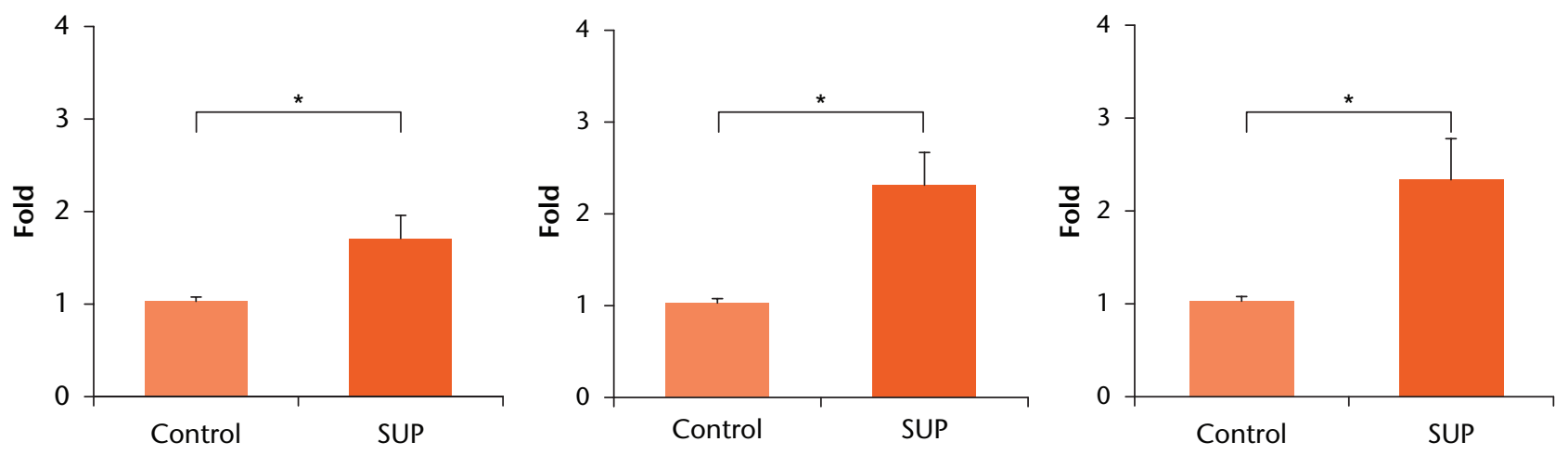

Fig. 3b

Osteogenic differentiation capacity: a) Photomicrographs showing alizarin red staining at 14 days after addition of skeletal muscle supernatant. Control cultures were treated with PBS: top row: supernatant group; bottom row: control group; b) expression levels of RUNX2 (left), COL1a1 (middle) and osteocalcin (right) at 14 days after addition of skeletal muscle supernatant, analysed by real-time PCR $(n=6)(* ; p<0.05)$.

surgical site. Therefore, we considered that skeletal muscle would be a feasible autologous candidate tissue for the point-of-care approach.

In our study, muscle supernatant could not only promote cell proliferation, but also accelerate osteogenic differentiation of MSCs and angiogenesis of HUVECs, which suggests that muscle supernatant produced by a simple, easy and rapid method has effects on tissue repair and regeneration. In particular, bFGF is recognised as playing an important role in osteogenesis and angiogenesis. Many studies suggest that bFGF contributes to the repair and regeneration of a variety of injured tissues including bone, ${ }^{17-19}$ cartilage, $^{18}$ nerve tissue, ${ }^{19}$ and wound healing. 20,21 In the current study, we confirmed the abundant content of bFGF in muscle supernatant (325.6 pg/mL, SD 210.2). According to previous reports, PRP contains smaller amounts of bFGF $(37.9 \mathrm{pg} / \mathrm{mL}$, SD $7.8),{ }^{22}$ a fact which supports the suggestion that skeletal muscle supernatant may have superior biological properties compared with PRP.

Regarding another aspect of skeletal muscle supernatant, it was confirmed that a number of cells, such as CD34+ and CD44+/CD105+, exist in the supernatant. CD34+ cells are commonly known not only as haematopoietic stem/progenitor cells, but also as endothelial or osteogenic progenitor cells, and this fraction has 

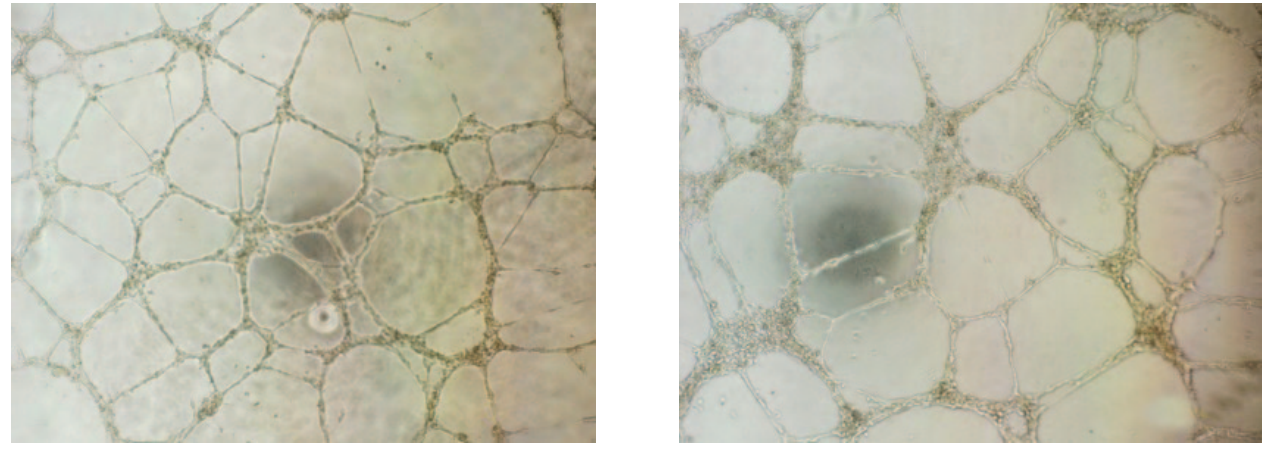

Fig. $4 a$
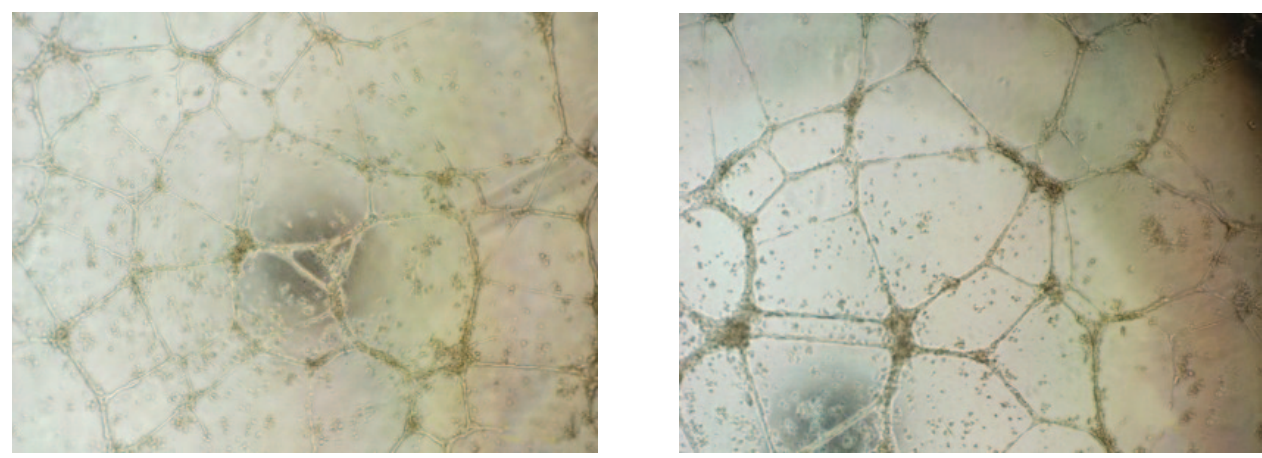

Fig. $4 \mathrm{~b}$
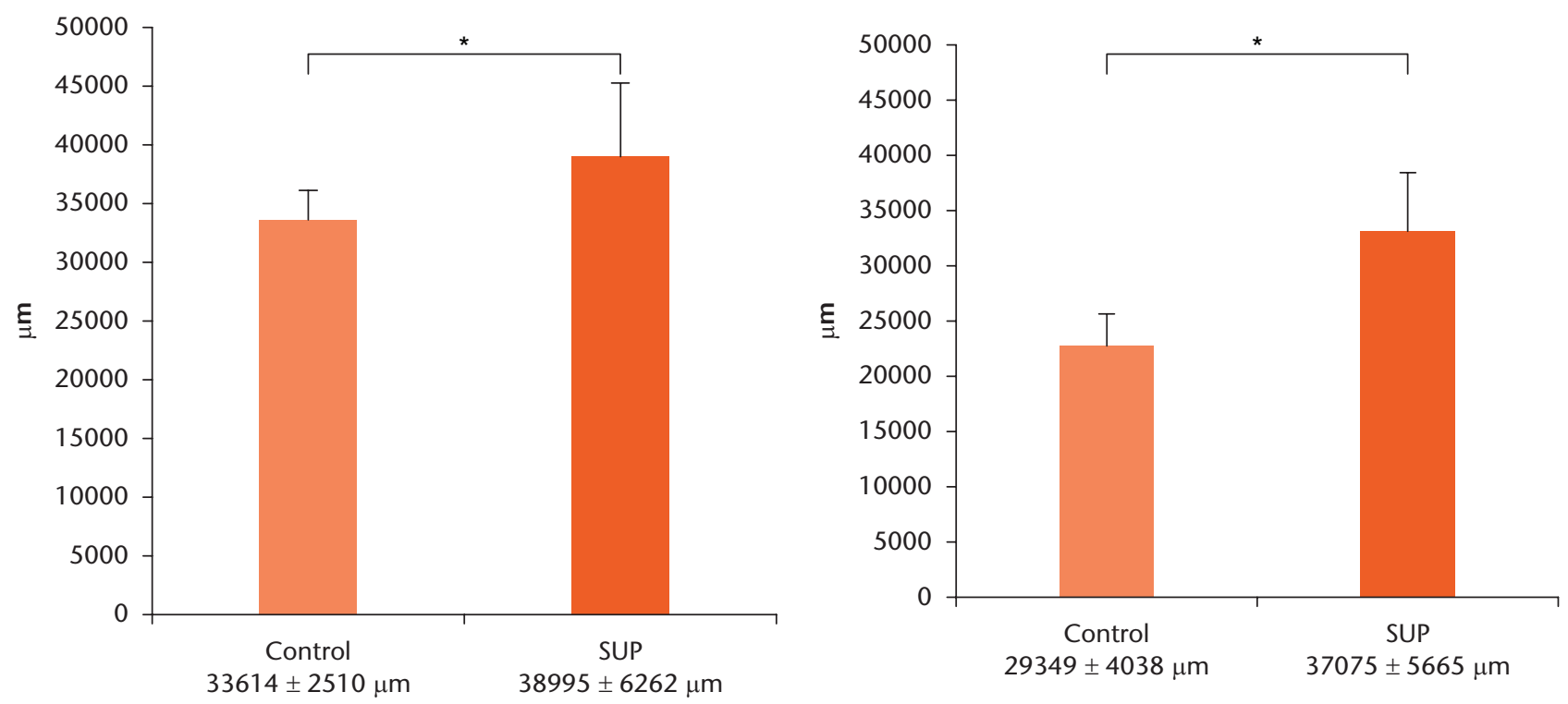

Fig.4c

Photomicrographs of tube formation in cultures of HUVECs at 12 (a) and 24 hours (b) after addition of skeletal muscle supernatant. Control cultures were treated with PBS: (a) supernatant group at 12 hours (left) and control group at 12 hours (right); (b) supernatant group at 24 hours (left) and control group at 24 hours (right). Total tube length measured at 12 (left) and 24 hours (right) after addition of skeletal muscle supernatant (c) ( $n=6$ ) ( $*$; < 0.05$)$. The control group was treated with $5 \mu \mathrm{L}$ PBS and the supernatant group was treated with $5 \mu \mathrm{L}$ of muscle supernatant $(n=6)$. Data are expressed as mean and standard deviation (*; $\mathrm{p}<0.05)$.

great therapeutic potential for tissue repair via angiogenesis in vivo. ${ }^{23}$ Although the populations of these cell fractions are very limited in the supernatant, their beneficial effect at the administered site would still be evident due to their naturally limited frequency in the human body.

As demonstrated in this study, skeletal muscle tissue can be obtained from the semitendinosus tendon. 
Therefore, the surgical reconstruction of the $A C L$, especially using autologous hamstring tendon, would be a favourable clinical target of this point-of-care approach. Repair of ACL injury still involves several problems such as combined injuries of cartilage and meniscus, ${ }^{24,25}$ healing of the tendon-bone interface, ${ }^{26,27}$ and maturation of grafted tendon. ${ }^{28}$ To accelerate tissue repair and maturation, muscle tissue supernatant could be a promising option. Based on the biological properties of muscle supernatant, this could be applicable to various surgeries such as repair of fractures, tendon injuries, and wounds.

There are several limitations to this study. Firstly, the effective amounts of CKs and GFs or stem/progenitor cells necessary for tissue repair is still unclear. The obtainable volume of muscle is limited. In our study, the average amount of semitendinosus muscle harvested was approximately $1.5 \mathrm{~g}$, and this yielded approximately 3.0 $\mathrm{mL}$ of muscle supernatant, which contained approximately 0.9 ng bFGF. The application of muscle supernatant would be mainly for local use such as in meniscus tear and cartilage defects. Hence, this approach might lead to successful results when used for tissue repair and regeneration depending on the administration site, although the volume of factors would be very limited. Second, muscle supernatant may contain factors with the adverse effects of exposure to proinflammatory cytokines. Our data demonstrated that muscle supernatant in large quantities could accelerate cell proliferation, osteogenesis and angiogenesis. The effect on chondrogenesis, neurogenesis, and tendon healing should also be examined to confirm its universal effect. Moreover, the orchestration of CKs and GFs that we presented in this study has to be investigated. Finally, the therapeutic potential in vivo was not analysed in animal studies. Further investigation will be needed before future clinical application can be undertaken.

In conclusion, the current study presents the potential of a new point-of-care strategy for regenerative medicine using skeletal muscle supernatant. This attractive approach and material could be a promising option not only for use in knee surgery but also in other tissue repair and regeneration procedures in the clinical setting.

\section{References}

1. Murray IR, Corselli M, Petrigliano FA, Soo C, Péault B. Recent insights into the identity of mesenchymal stem cells: Implications for orthopaedic applications. Bone Joint J 2014:96-B:291-298.

2. Farr J, Gomoll AH. 2016 barriers to cartilage restoration. J Clin Orthop Trauma 2016;7:183-186.

3. Jankowski RJ, Deasy BM, Huard J. Muscle-derived stem cells. Gene Ther 2002;9:642-647.

4. Deasy BM, Jankowski RJ, Huard J. Muscle-derived stem cells: characterization and potential for cell-mediated therapy. Blood Cells Mol Dis 2001;27:924-933.

5. Drummond MJ. MicroRNAs and exercise-induced skeletal muscle adaptations. J Physiol 2010;588:3849-3850.

6. Adachi N, Sato K, Usas A, et al. Muscle derived, cell based ex vivo gene therapy for treatment of full thickness articular cartilage defects. J Rheumato/ 2002;29:1920-1930.

7. Gussoni E, Soneoka Y, Strickland CD, et al. Dystrophin expression in the $\mathrm{mdx}$ mouse restored by stem cell transplantation. Nature 1999;401:390-394.
8. Lee JY, Qu-Petersen Z, Cao B, et al. Clonal isolation of muscle-derived cells capable of enhancing muscle regeneration and bone healing. $J$ Cell Biol 2000;150:1085-1100.

9. McCarthy HD, Samani-Radia D, Jebb SA, Prentice AM. Skeletal muscle mass reference curves for children and adolescents. Pediatr Obes 2014;9:249-259.

10. Gregory CA, Gunn WG, Peister A, Prockop DJ. An Alizarin red-based assay of mineralization by adherent cells in culture: comparison with cetylpyridinium chloride extraction. Anal Biochem 2004;329:77-84.

11. Gholobova D, Decroix L, Van Muylder V, et al. Endothelial Network Formation Within Human Tissue-Engineered Skeletal Muscle. Tissue Eng Part A 2015;21:2548-2558.

12. Catelas I, Dwyer JF, Helgerson S. Controlled release of bioactive transforming growth factor beta-1 from fibrin gels in vitro. Tissue Eng Part C Methods 2008;14:119-128.

13. Trombi L, Mattii L, Pacini S, et al. Human autologous plasma-derived clot as a biological scaffold for mesenchymal stem cells in treatment of orthopedic healing. $J$ Orthop Res 2008;26:176-183.

14. Schildhauer T, Seybold D, Geßmann J, et al. Fixation of porous calcium phosphate with expanded bone marrow cells using an autologous plasma clot. Materia/wissenschaft und Werkstofftechnik 2007;38:1012-1014.

15. Alsousou J, Thompson M, Hulley P, et al. The biology of platelet-rich plasma and its application in trauma and orthopaedic surgery: a review of the literature. J Bone Joint Surg [Br]2009;91-B:987-996.

16. Henning CE, Lynch MA, Yearout KM, et al. Arthroscopic meniscal repair using an exogenous fibrin clot. Clin Orthop Relat Res 1990;252:64-72.

17. Frenkel SR, Grande DA, Collins M, Singh IJ. Fibroblast growth factor in chick osteogenesis. Biomaterials 1990;11:38-40.

18. Frenkel SR, Herskovits MS, Singh IJ. Fibroblast growth factor: effects on osteogenesis and chondrogenesis in the chick embryo. Acta Anat (Basel) 1992;145:265-268.

19. Mudò G, Bonomo A, Di Liberto V, et al. The FGF-2/FGFRs neurotrophic system promotes neurogenesis in the adult brain. J Neural Transm (Vienna)2009;116:995-1005.

20. Werner S, Grose R. Regulation of wound healing by growth factors and cytokines. Physiol Rev 2003;83:835-870.

21. Greenhalgh DG, Sprugel KH, Murray MJ, Ross R. PDGF and FGF stimulate wound healing in the genetically diabetic mouse. Am J Pathol 1990;136:1235-1246.

22. Yang HS, Shin J, Bhang SH, et al. Enhanced skin wound healing by a sustained release of growth factors contained in platelet-rich plasma. Exp Mol Med 2011;43:622-629

23. Matsumoto T, Kawamoto A, Kuroda R, et al. Therapeutic potential of vasculogenesis and osteogenesis promoted by peripheral blood CD34-positive cells for functional bone healing. Am J Pathol 2006;169:1440-1457

24. Slauterbeck JR, Kousa P, Clifton BC, et al. Geographic mapping of meniscus and cartilage lesions associated with anterior cruciate ligament injuries. J Bone Joint Surg [Am]2009;91-A:2094-2103.

25. Ralles S, Agel J, Obermeier M, Tompkins M. Incidence of secondary intraarticular injuries with time to anterior cruciate ligament reconstruction. Am J Sports Med 2015;43:1373-1379.

26. Rodeo SA, Kawamura S, Kim HJ, et al. Tendon healing in a bone tunnel differs at the tunnel entrance versus the tunnel exit: an effect of graft-tunnel motion? Am J Sports Med 2006;34:1790-1800.

27. Weber $\mathbf{A E}$, Delos $\mathbf{D}$, Oltean $\mathbf{H N}$, et al. Tibial and femoral tunnel changes after ACL reconstruction: a prospective 2-year longitudinal MRI study. Am J Sports Med 2015;43:1147-1156

28. Pauzenberger L, Syré S, Schurz M. "Ligamentization" in hamstring tendon grafts after anterior cruciate ligament reconstruction: a systematic review of the literature and a glimpse into the future. Arthroscopy 2013;29:1712-1721.

Funding Statement

This work was carried out at the Analysis Center of Life Science, Natural Science Center for Basic Research and Development, Hiroshima University.

Author Contribution

M. Yoshikawa: Acquisition of data, Analysis and interpretation of data, Drafting of manuscript.

T. Nakasa: Study conception and design, Critical revision of the article for importan intellectual content.

M. Ishikawa: Acquisition of data.

N. Adachi: Final approval of the article.

M. Ochi: Final approval of the article.

ICMJE COI Statement

None declared.

() 2017 Nakasa et al. This is an open-access article distributed under the terms of the Creative Commons Attributions licence (CC-BY-NC), which permits unrestricted use, distribution, and reproduction in any medium, but not for commercial gain, provided the original author and source are credited. 